\title{
Factors Associated with Coronavirus 2019 (COVID-19) Prevention Behaviors among Health Sciences Students of a Higher Education Institution in Yala Province, Thailand
}

\author{
Awirut SINGKUN*, Piriya PATIWIKRIWONG, \\ Khajornsak CHAINAPONG and Phakkhanat WEERAKHACHON
}

\begin{abstract}
Sirindhorn College of Public Health, Yala, Faculty of Public Health Sciences and Allied Health Sciences, Praboromarajchanok Institute, Yala 95000, Thailand
\end{abstract}

('Corresponding author's e-mail: awirut@yala.ac.th)

Received: 28 April 2020, Revised: 29 July 2020, Accepted: 6 August 2020

\begin{abstract}
The objectives of this research were to study the knowledge on COVID-19 infection, satisfaction of the measures on disease prevention and control, and the association between factors and COVID-19 prevention behaviors among health sciences students. Content validity and reliability of research tools were measured. Ethical for human study was approved by Research Ethic Committee. Self-administered questionnaires were used to collect the data of 184 health sciences students in April, 2020. First, a proportion by curriculum and year of study was made. Then, simple random sampling was created based on student identification. Data analysis involved descriptive statistics, Chi-square and Fisher's exact tests. The results found that participants had correct answer for COVID-19 infection (70.65 - 99.46 \%) and had a good knowledge level on COVID-19 infection (90.22\%). Their satisfaction of the institute's measures on COVID-19 prevention and control was at High level (50.54\%). COVID-19 prevention behavior among participants was at moderate level (51.63\%). Age, year level, payment per week, and satisfaction level of their organization's measures on COVID-19 prevention and control were closely associated with COVID-19 prevention behavior $(p-$ value $<0.05)$. These results could be used as guidelines to arrange additional activities for students in lower age and integrate health concern into the curriculum in early year of study. For further study, satisfaction theory can be applied to encourage students to have positive behaviors.
\end{abstract}

Keywords: Coronavirus, COVID-19, Knowledge, Prevention behavior, Higher education institute

\section{Introduction}

Coronavirus Disease 2019 (COVID-19) is a disease caused by severe acute respiratory syndrome coronavirus 2 (SARS-CoV-2); it was identified as the causative agent for a cluster of pneumonia cases. The first official reported of COVID-19 infection patient was found in Wuhan City, Hubei province, China in December, 2019 [1]. The median time from illness onset to invasive mechanical ventilation was 14 - 15 days. The clinical spectrum of this infection appears to be wide, encompassing asymptomatic infection, mild upper respiratory tract illness [2]. The most commonly reported symptoms including fever (98\%), dry cough (76\%) [3], and shortness of breath, and most patients (80\%) experienced mild illness [4], myalgia or fatigue (44\%), less common symptoms were sputum production $(28 \%)$, headache $(8 \%)$, hemoptysis $(5 \%)$, and diarrhea $(3 \%)$. Dyspnea developed up to $55 \%$ of patients. All patients had pneumonia with abnormal findings on chest CT. Complications included acute respiratory distress syndrome (29\%), acute cardiac injury (12\%) and secondary infection (10\%) [3]. About $14 \%$ of patients experienced severe disease and $5 \%$ were critically ill [4] and severe viral pneumonia with respiratory 
http://wjst.wu.ac.th

failure and even death, with many patients being hospitalized with pneumonia. Sepsis was the most frequently observed complication, followed by respiratory failure, acute respiratory distress syndrome (ARDS), heart failure, and septic shock [2]. At least 2 studies confirmed that increased age was associated with death in patients with COVID-19. So, the potential risk factor was older age ( $>60$ years old) $[2,4]$ and co-morbid disease. Mortality in those with critical illness has been reported as over $50 \%$ [4].

The rise in the number of patients who are infected and those who require intensive care admittance will seemingly continue to soar exponentially. If the change in the slope of the curve does not take place soon, the clinical and social problems will take on unmanageable dimensions, which are expected to have catastrophic results [5].

More than 210 Countries and Territories around the world have reported a total of 2,996,563 confirmed cases of COVID-19, and a death toll of 207,023 deaths [6]. The spread of COVID-19 infection has already taken on pandemic proportions. A global response to prepare health systems worldwide is imperative [5].

In Thailand, $26^{\text {th }}$ April 2020, 15 new cases of laboratory-confirmed COVID-19 were announced by the Ministry of Public Health of Thailand, bringing the total number of 2,922 cases. Of these, $88 \%(2,594$ cases) have recovered, about $1.8 \%$ (51 cases) have died, and $9.5 \%$ (277 cases) are receiving treatment [7].

There are the notifications of the Ministry of Health on COVID-19 about the countries where are dangerous areas of communicable diseases. These countries are Republic of Korea, People's Republic of China (including Macao and Hong Kong), Italian Republic, Islamic Republic of Iran [8] Malaysia, Kingdom of Cambodia, Lao People's Democratic Republic, Republic of Indonesia, and Republic of the Union of Myanmar. All people came back from these countries will be quarantined in a location by the government for at least 14 days. Violation or defiance is punishable by law. Penalty includes fine, imprisonment or both [9].

Basic measures that will be implemented by all provinces, regardless of level of risk, are promoting health literacy on prevention and control of COVID-19, including personal hygiene, active case detection and isolation of case, tracing and quarantining their contacts protection of vulnerable groups such as the elderly those with chronic diseases, healthcare facility preparedness, including enhanced infection prevention and control, increasing social distancing [7].

In the late of March 2020, COVID-19 pandemic affected to health personnel in Bannang Sata Hospital, Yala province. The infected cases were one physician, 2 nurses and approximately twenty hospital staff were being quarantined. This situation caused of temporarily closed, except for emergency case only [10]. In April 2020, 40 patients of COVID-19 infection were confirmed. It was the highest number of cases found that confirmed by the Center for COVID-19 Situation Administration. More than half of these patients came back from religious activities in neighboring countries as well as religious events in the province and other provinces. [10,11]. To control spreading of COVID-19, the 3 deep southern provinces has announced to lockdown the area to local outbreak and restricted the movements of people across province $[10,12,13]$. These 3 provinces, Yala has the highest accumulated COVID-19 patients-Yala has 121 cases, Pattani 91 cases and Narathiwat 33 cases [14].

A Higher Education Institution on health sciences in Yala province of Thailand has a safety college policy. Especially in COVID-19 pandemic era, all personnel and students were supported face masks, alcohol spray (it can be refilled) liquid soap at all toilets and sinks from the college, in order to protect themselves. These supports may elevate their satisfaction towards the organization measures and make them have more participation on health protection and prevention from the disease. However, because of the novel disease, there was no study about COVID-19 prevention behaviors in health sciences students. In addition, health sciences students must be a good role model to protect themselves from COVID-19 infection. It was necessary to assess their knowledge on COVID-19. So, the study of COVID-19 prevention behaviors among health sciences students was studied to explore their knowledge on COVID19 infection and satisfaction of the measures of institute's on disease prevention and control and find out the association between the studied factors and student's behavior on COVID-19 infected prevention. 


\section{Materials and methods}

\section{Study design}

This descriptive cross-sectional study was performed between January and April, 2020 and the data collection was conducted in April, 2020.

\section{Study procedure}

The population were the students registered in the second semester of the academic year of 20192020 (December, 2019 - April, 2020) and not the subject of community health practice, with the total of 329 students. The sample size was at least 178 students calculated by using the Krejcie and Morgan's formula [15];

$$
n=\frac{\chi^{2} N p(1-p)}{e^{2}(N-1)+\chi^{2} p(1-p)}
$$

$\mathrm{n}=$ sample size

$\mathrm{N}=$ population size

e $=$ acceptable sampling error

$\chi 2=$ chi-square of degree of freedom 1 and confidence $95 \%(=3.841)$

$\mathrm{p}=$ proportion of population (if unknown $=0.5$ )

$$
\begin{aligned}
& =\frac{3.841 \times 329 \times 0.05 \times(1-0.5)}{\left((0.05)^{2} \times(329-1)\right)+(3.841 \times 0.5 \times(1-0.5))} \\
& =177.48 \sim 178
\end{aligned}
$$

The sample size was at least 178 students. Researcher added 5\% participants in order to avoid incomplete questionnaires. Making a proportion was done by the curriculum and year level of the study. Then, simple random sampling was created based on student identification. Participants felt free to answer the questionnaires at private area and could submit the questionnaires by themselves in the data collecting box. This way, the process remained confidential and participant's identity was kept unidentified from outsiders. The total response was 184 participants for this study.

The inclusion criteria:

1. Full time health sciences students in the $2^{\text {nd }}$ semester of academic year of 2019 in a higher education institute.

2. Consent to participate in this study.

The exclusion criterion was incomplete questionnaire.

\section{Materials}

The questionnaires were made by researchers from reviewing the body of the literature. Selfadministered questionnaires were used for the data collection that consisted of 4 parts.

Part I: General information had 9 items.

Part II: Knowledge on COVID-19 infection had 16 items, in which participants answered yes, no, or not sure. Then, conversed these responses into category data (true or false answer). Finally, interpretations are divided into 3 levels (good, moderate, and poor level) according to Bloom's criterion cut point [16].

Knowledge level
Good
Moderate
Poor

\section{Score}

$12.80-16.00$ points

$9.60-12.79$ points

Less than 9.60 points 
http://wjst.wu.ac.th

Part III: Satisfaction of the measures on disease prevention and control had 7 items (most, much, medium, little, or least). Interpretations of the measures' satisfaction are categorized into 3 levels, according to class interval. Satisfaction level were high, moderate, and low level.

$\begin{array}{lc}\text { Satisfaction level } & \text { Score } \\ \text { High } & 25.68-35.00 \text { points } \\ \text { Moderate } & 16.33-25.67 \text { points } \\ \text { Low } & 7.00-16.32 \text { points }\end{array}$

Part IV: COVID-19 prevention behavior had 21 items. Eleven items were time assessment (every time, 4 out of 5 times, 2 - 3 out of 5 times, 1 out of 5 times, or never) and remain questions were daily assessment (everyday, 5 - 6 days/week, 3 - 4 days/week, 1 - 2 days/week, or never). Prevention behaviors were divided into 3 levels according to class interval including good, moderate, and poor level.

$\begin{array}{lc}\text { Prevention behavior level } & \text { Score } \\ \text { High } & 78.00-105.00 \text { points } \\ \text { Moderate } & 49.00-77.00 \text { points } \\ \text { Low } & 21.00-48.00 \text { points }\end{array}$

Content validity was approved by 3 public health academicians. The Index of Item-Objective Congruence (IOC) of Part I was 1.00, Part II, IOC ranged from 0.67 - 1.00, Part III and Part IV, IOC were 1.00. The reliability of the knowledge on COVID-19 infection was analyzed by using $\mathrm{KR}_{20}$; the coefficient was 0.816 .Cronbach's $\boldsymbol{\alpha}$ coefficient was used to test for the measures of satisfaction on disease prevention and control; $\boldsymbol{\alpha}$ coefficient was 0.941 .

\section{Data analysis}

Frequency and percentage were used to analyze category data of each part and the association between variables and COVID-19 prevention behavior were analyzed by Chi-square and Fisher's exact test.

\section{Ethical consideration}

Ethical consideration and approval were made by the Ethic Committee for Human Research Subjects of Sirindhorn College of Public Health, Yala (Project code: 082 - 63)

\section{Results and discussion}

Most of the participants were female (94.57\%); most of them were at the age of 20 years old and older $(63.59 \%)$, studying in Bachelor of Public Health (Dental Public Health) $(28.26 \%), 1^{\text {st }}$ year level $(43.48 \%)$, staying in college dormitory $(83.15 \%)$, coming from the Southern region of Thailand (apart from the 3 southernmost provinces) (68.48\%). Their payment per week lower than 1,000 Baht and 1,000 - 1,500 Baht were $47.83 \%$ and $44.57 \%$, respectively (Table 1). 
http://wjst.wu.ac.th

Table 1 General information $(n=184)$.

\begin{tabular}{|c|c|c|}
\hline General information & Frequency & $\%$ \\
\hline \multicolumn{3}{|l|}{ Sex } \\
\hline Male & 10 & 5.43 \\
\hline Female & 174 & 94.57 \\
\hline \multicolumn{3}{|l|}{ Age $(\max 26.17, \min 18.25$, mean 20.60 years $)$} \\
\hline Lower than 20 years & 67 & 36.41 \\
\hline 20 years and upper & 117 & 63.59 \\
\hline \multicolumn{3}{|l|}{ Curriculum } \\
\hline Diploma of Public Health (Pharmacy technique) & 33 & 17.93 \\
\hline \multicolumn{3}{|l|}{ Dip. of P.H. (Pharmacy technique) } \\
\hline Diploma of Science Program in Emergency Medical Operation & 17 & 9.24 \\
\hline \multicolumn{3}{|l|}{ Dip. (Emergency Medical Operation) } \\
\hline Bachelor of Public Health (Community Health) & 42 & 22.83 \\
\hline \multicolumn{3}{|l|}{$\begin{array}{l}\text { B.P.H. Community Health } \\
\text { B. }\end{array}$} \\
\hline Bachelor of Public Health (Dental Public Health) & 52 & 28.26 \\
\hline \multicolumn{3}{|l|}{ B.P.H. Dental Public Health } \\
\hline \multicolumn{3}{|l|}{ B.TM. } \\
\hline \multicolumn{3}{|l|}{ Curriculum level } \\
\hline Diploma & 50 & 27.17 \\
\hline Bachelor & 134 & 72.83 \\
\hline \multicolumn{3}{|l|}{ Year level } \\
\hline $1^{\text {st }}$ year & 80 & 43.48 \\
\hline $2^{\text {nd }}$ year & 55 & 29.89 \\
\hline $3^{\text {rd }}$ year & 49 & 26.63 \\
\hline \multicolumn{3}{|l|}{ Residence } \\
\hline Dormitory & 153 & 83.15 \\
\hline Outside & 31 & 16.85 \\
\hline \multicolumn{3}{|l|}{ Home } \\
\hline Three southernmost provinces & 58 & 31.52 \\
\hline Other provinces & 126 & 68.48 \\
\hline \multicolumn{3}{|l|}{ Payment per week } \\
\hline Lower than 1,000 Baht & 88 & 47.83 \\
\hline $1,000-1,500$ Baht & 82 & 44.57 \\
\hline More than 1,500 Baht & 14 & 7.61 \\
\hline
\end{tabular}

The results showed the participants' knowledge on COVID-19 infection. The first 3 items that they had correct answers were washing hands with soap or alcoholic gel can prevent the risk of COVID-19 infection $(99.46 \%)$. Second, gathering or crowded people can cause both infection from others and distribution to others $(98.91 \%)$. Third, COVID-19 patients should be quarantined (97.83\%). For incorrect answer, the first 3 items were there is no risk country for COVID-19 infection, but it is the political or economic reason to promote tourism in their own country $(29.35 \%)$. Second, chronic disease patients have more chance for COVID-19 infection $(25.54 \%)$. Third, wearing face mask, will not be infected by COVID-19 (21.20\%), as indicated in Table 2. Converse participant's knowledge was split into 3 levels and showed that they had good knowledge on COVID-19 infection (90.22\%), as seen in Table 3. 
Table 2 Knowledge on COVID-19 infection $(n=184)$.

\begin{tabular}{|c|c|c|c|c|}
\hline \multirow{2}{*}{ Statement } & \multicolumn{2}{|c|}{ True } & \multicolumn{2}{|c|}{ False } \\
\hline & Frequency & $(\%)$ & Frequency & $(\%)$ \\
\hline $\begin{array}{l}\text { 1. Close contact to patient may cause of COVID-19 } \\
\text { infection }\end{array}$ & 151 & $(82.07)$ & 33 & (17.93) \\
\hline $\begin{array}{l}\text { 2. Touch on your face or eyes could be the risk of } \\
\text { COVID-19 infection }\end{array}$ & 168 & (91.30) & 16 & $(8.70)$ \\
\hline $\begin{array}{l}\text { 3. Close to patient with cough or sneeze could be the } \\
\text { risk of COVID-19 infection }\end{array}$ & 160 & $(86.96)$ & 24 & (13.04) \\
\hline $\begin{array}{l}\text { 4. COVID-19 immediately die after ran out of } \\
\text { patient's body* }\end{array}$ & 153 & (83.15) & 31 & (16.85) \\
\hline $\begin{array}{l}\text { 5. You should see the doctor when you have fever, } \\
\text { cough or respiratory distress after contacted to } \\
\text { influenza liked patients }\end{array}$ & 174 & (94.57) & 10 & $(5.43)$ \\
\hline $\begin{array}{l}\text { 6. Wearing a face mask, lowering the risk of } \\
\text { COVID-19 infection }\end{array}$ & 179 & (97.28) & 5 & $(2.72)$ \\
\hline $\begin{array}{l}\text { 7. Patient who wear a face mask could decrease } \\
\text { COVID-19 distribution }\end{array}$ & 175 & (95.11) & 9 & $(4.89)$ \\
\hline $\begin{array}{l}\text { 8. *People will not be infected by COVID-19 if they } \\
\text { wear a face mask }\end{array}$ & 145 & $(78.80)$ & 39 & $(21.20)$ \\
\hline $\begin{array}{l}\text { 9. Washing hands with soap or alcoholic gel, } \\
\text { preventing the risk of COVID-19 infection }\end{array}$ & 183 & (99.46) & 1 & $(0.54)$ \\
\hline $\begin{array}{l}\text { 10. COVID-19 patients should be home and avoid } \\
\text { going to public places }\end{array}$ & 180 & $(97.83)$ & 4 & $(2.17)$ \\
\hline $\begin{array}{l}\text { 11. Cleaning your hands before touching to your } \\
\text { eyes, nose, mouth, or face could decrease the risk } \\
\text { of COVID-19 infection }\end{array}$ & 175 & (95.11) & 9 & (4.89) \\
\hline $\begin{array}{l}\text { 12. *There is no COVID-19 risk country, but it is the } \\
\text { political or economic reason to promote tourism } \\
\text { in their own country }\end{array}$ & 130 & $(70.65)$ & 54 & (29.35) \\
\hline $\begin{array}{l}\text { 13. The risk of COVID-19 infection will increase if } \\
\text { you share your clothes with patient }\end{array}$ & 171 & (92.93) & 13 & $(7.07)$ \\
\hline $\begin{array}{l}\text { 14. Chronic disease patients have more susceptible to } \\
\text { COVID-19 infection }\end{array}$ & 137 & (74.46) & 47 & $(25.54)$ \\
\hline $\begin{array}{l}\text { 15. Activities of gathering or crowded people can } \\
\text { cause both infection from others and distribution } \\
\text { to others }\end{array}$ & 182 & (98.91) & 2 & (1.09) \\
\hline $\begin{array}{l}\text { 16. Closed environments - bus, aircraft - have more } \\
\text { chance for COVID-19 infection than ventilated } \\
\text { area }\end{array}$ & 177 & $(96.20)$ & 7 & $(3.80)$ \\
\hline
\end{tabular}

*False statements 
http://wjst.wu.ac.th

Table 3 Knowledge level on COVID-19 infection $(n=184)$.

\begin{tabular}{lcc}
\hline \multicolumn{1}{c}{ Knowledge level } & Frequency & \% \\
\hline Good (12.80 - 16.00 points) & 166 & 90.22 \\
Moderate (9.60 - 12.79 points) & 14 & 7.61 \\
Poor (lower than 9.60 points) & 4 & 2.17 \\
\hline
\end{tabular}

Total score 16.00, max. 16.00, min. 1.00, mean 14.33, SD. 1.95

Table 4 showed the participants' satisfaction of their institute's measures on disease prevention and control. Their most satisfaction were alcohol spray supported $(28.80 \%)$, liquid soap and hand wash gel provided $(20.11 \%)$ and providing alcohol spray and alcohol for using that equal to screening test for the risk of COVID-19 infection (18.48\%). Their satisfaction was in high level $50.54 \%$ followed by medium level $46.74 \%$ (Table 5).

Table 4 Satisfaction of the measures on disease prevention and control $(n=184)$.

\begin{tabular}{lccccc}
\hline \multicolumn{1}{c}{ Statement } & \multicolumn{4}{c}{ Satisfaction $\mathrm{n}(\%)$} \\
\cline { 2 - 6 } & Most & Much & Medium & Little & Least \\
\hline 1. Teaching and learning about COVID-19 & 33 & 101 & 48 & 0 & 2 \\
transmission, prevention, and control & $(17.93)$ & $(54.89)$ & $(26.09)$ & $(00.00)$ & $(1.09)$ \\
2. Supporting alcohol spray to students and all & 53 & 80 & 41 & 9 & 1 \\
personnel & $(28.80)$ & $(43.48)$ & $(22.28)$ & $(4.89)$ & $(0.54)$ \\
3. Supplying liquid soap and hand wash gel at & 37 & 76 & 53 & 14 & 4 \\
basin and sinks & $(20.11)$ & $(41.30)$ & $(28.80)$ & $(7.61)$ & $(2.17)$ \\
4. Providing alcohol spray and alcohol gel in the & 34 & 67 & 70 & 12 & 1 \\
college area & $(18.48)$ & $(36.41)$ & $(38.04)$ & $(6.52)$ & $(0.54)$ \\
5. Cleaning public equipment and all college area & 28 & 49 & 79 & 26 & 1 \\
& $(15.30)$ & $(26.78)$ & $(43.17)$ & $(14.21)$ & $(0.55)$ \\
6. Supporting face mask to students and all & 33 & 64 & 56 & 27 & 4 \\
personnel & $(17.93)$ & $(34.78)$ & $(30.43)$ & $(14.67)$ & $(2.17)$ \\
7. Screening test for the risk of COVID-19 & 34 & 76 & 49 & 18 & 7 \\
infection among students, all personnel, and & $(18.48)$ & $(41.30)$ & $(16.63)$ & $(9.78)$ & $(3.80)$ \\
arrived person from community practice & & & & & \\
\hline
\end{tabular}

Table 5 Satisfaction level of disease prevention and control measures $(n=184)$.

\begin{tabular}{lcc}
\hline \multicolumn{1}{c}{ Satisfaction level } & Frequency & \% \\
\hline High $(25.68-35.00$ points $)$ & 93 & 50.54 \\
Moderate $(16.33-25.67$ points $)$ & 86 & 46.74 \\
Low $(7.00-16.32$ points $)$ & 5 & 2.72 \\
\hline
\end{tabular}

Total score 35.00, max. 35.00, min. 15.00, mean 25.72, S.D. 5.02

The participants' behaviors on COVID-19 prevention between February and March, 2020 were as follow. Behaviors defined as times to do, they reported of every time doing - they wear a face mask when they closed to other people or go to public area $(64.84 \%)$, and they immediate sip or drink water when they feel dry or thirsty $(50.55 \%)$. For daily behaviors, they emphasized on food that they eat hot meal or newly cooked food (37.91\%), and they have sufficient amount of meals each day (37.02\%) (Table 6). Their prevention behavior was in medium level $51.63 \%$ and high level $46.74 \%$ whereas little percentage was in low level $1.63 \%$ (Table 7). 
http://wjst.wu.ac.th

Table 6 COVID-19 prevention behavior (During the past 2 months; February and March, 2020) $(n=$ 184).

\begin{tabular}{|c|c|c|c|c|c|}
\hline \multirow[b]{2}{*}{ Statement } & \multicolumn{5}{|c|}{ Frequency of behavior $n(\%)$} \\
\hline & 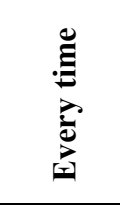 & 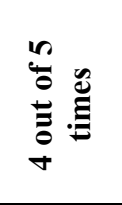 & 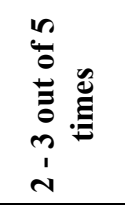 & 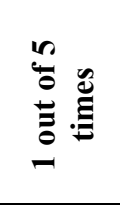 & 离 \\
\hline $\begin{array}{l}\text { 1. You wash your hand with liquid soap or alcohol gel } \\
\text { before eating }\end{array}$ & $\begin{array}{c}58 \\
(31.52)\end{array}$ & $\begin{array}{c}60 \\
(32.61)\end{array}$ & $\begin{array}{c}47 \\
(25.54)\end{array}$ & $\begin{array}{c}18 \\
(9.78)\end{array}$ & $\begin{array}{c}1 \\
(0.54)\end{array}$ \\
\hline $\begin{array}{l}\text { 2. You wash your hand with liquid soap or alcohol gel } \\
\text { after going to restroom }\end{array}$ & $\begin{array}{c}74 \\
(40.22)\end{array}$ & $\begin{array}{c}50 \\
(27.17)\end{array}$ & $\begin{array}{c}54 \\
(29.35)\end{array}$ & $\begin{array}{c}5 \\
(2.72)\end{array}$ & $\begin{array}{c}1 \\
(0.54)\end{array}$ \\
\hline $\begin{array}{l}\text { 3. You wash your hand with liquid soap or alcohol gel } \\
\text { when you touched other people's clothes }\end{array}$ & $\begin{array}{c}40 \\
(21.74)\end{array}$ & $\begin{array}{c}61 \\
(33.15)\end{array}$ & $\begin{array}{c}60 \\
(32.61)\end{array}$ & $\begin{array}{c}19 \\
(10.33)\end{array}$ & $\begin{array}{c}4 \\
(2.17)\end{array}$ \\
\hline $\begin{array}{l}\text { 4. You wash your hand with liquid soap or alcohol gel } \\
\text { after shake hand or contact to other people }\end{array}$ & $\begin{array}{c}36 \\
(19.57)\end{array}$ & $\begin{array}{c}69 \\
(37.50)\end{array}$ & $\begin{array}{c}54 \\
(29.35)\end{array}$ & $\begin{array}{c}23 \\
(12.50)\end{array}$ & $\begin{array}{c}2 \\
(1.09)\end{array}$ \\
\hline $\begin{array}{l}\text { 5. You wash your hand with liquid soap or alcohol gel } \\
\text { after using public equipment }\end{array}$ & $\begin{array}{c}67 \\
(36.41)\end{array}$ & $\begin{array}{c}69 \\
(37.50)\end{array}$ & $\begin{array}{c}38 \\
(20.65)\end{array}$ & $\begin{array}{c}8 \\
(4.35)\end{array}$ & $\begin{array}{c}2 \\
(1.09)\end{array}$ \\
\hline $\begin{array}{l}\text { 6. You clean public equipment with antiseptic or } \\
\text { alcohol spray before using }\end{array}$ & $\begin{array}{c}25 \\
(13.59)\end{array}$ & $\begin{array}{c}59 \\
(32.07)\end{array}$ & $\begin{array}{c}70 \\
(38.04)\end{array}$ & $\begin{array}{c}25 \\
(13.59)\end{array}$ & $\begin{array}{c}5 \\
(2.72)\end{array}$ \\
\hline $\begin{array}{l}\text { 7. When you feel dry or thirsty, you will immediate } \\
\text { sip or drink water }\end{array}$ & $\begin{array}{c}92 \\
(50.00)\end{array}$ & $\begin{array}{c}50 \\
(27.17)\end{array}$ & $\begin{array}{c}35 \\
(19.02)\end{array}$ & $(3.26)$ & $(0.54)$ \\
\hline $\begin{array}{l}\text { 8. You wear a face mask when you closed to other } \\
\text { people or go to public place }\end{array}$ & $\begin{array}{c}118 \\
(64.13)\end{array}$ & $\begin{array}{c}38 \\
(20.65)\end{array}$ & $\begin{array}{c}26 \\
(14.13)\end{array}$ & $\begin{array}{c}2 \\
(1.09)\end{array}$ & $\begin{array}{c}0 \\
(0.00)\end{array}$ \\
\hline $\begin{array}{l}\text { 9. If you can't avoid eating together, you and other } \\
\text { people use serving spoon }\end{array}$ & $\begin{array}{c}71 \\
(38.59)\end{array}$ & $\begin{array}{c}48 \\
(26.09)\end{array}$ & $\begin{array}{c}27 \\
(14.67)\end{array}$ & $\begin{array}{c}15 \\
(8.15)\end{array}$ & $\begin{array}{c}23 \\
(12.50)\end{array}$ \\
\hline 10. * You share equipment with your friends & $\begin{array}{l}18 \\
(9.78)\end{array}$ & $\begin{array}{c}27 \\
(14.67)\end{array}$ & $\begin{array}{c}33 \\
(17.93)\end{array}$ & $\begin{array}{c}42 \\
(22.83)\end{array}$ & $\begin{array}{c}64 \\
(34.78)\end{array}$ \\
\hline 11. * You share clothes or towel with your friends & $\begin{array}{c}6 \\
(3.26)\end{array}$ & $\begin{array}{c}12 \\
(6.52)\end{array}$ & $\begin{array}{c}15 \\
(8.15)\end{array}$ & $\begin{array}{c}12 \\
(6.52)\end{array}$ & $\begin{array}{c}139 \\
(75.54)\end{array}$ \\
\hline $\begin{array}{l}\text { 12. }{ }^{*} \text { You join the activities with unknown and } \\
\text { crowded people }\end{array}$ & $\begin{array}{c}8 \\
(4.35)\end{array}$ & $\begin{array}{c}26 \\
(14.13)\end{array}$ & $\begin{array}{c}49 \\
(26.63)\end{array}$ & $\begin{array}{c}73 \\
(39.67)\end{array}$ & $\begin{array}{c}28 \\
(15.22)\end{array}$ \\
\hline $\begin{array}{l}\text { 13. *You participate in college activities that many } \\
\text { people gather together }\end{array}$ & $\begin{array}{c}22 \\
(11.96)\end{array}$ & $\begin{array}{c}48 \\
(26.09)\end{array}$ & $\begin{array}{c}47 \\
(25.54)\end{array}$ & $\begin{array}{c}51 \\
(27.72)\end{array}$ & $\begin{array}{c}16 \\
(8.70)\end{array}$ \\
\hline $\begin{array}{l}\text { 14. *You contact to other people's equipment, bed, } \\
\text { clothes, or personal belongings }\end{array}$ & $\begin{array}{c}21 \\
(11.41)\end{array}$ & $\begin{array}{c}33 \\
(17.93)\end{array}$ & $\begin{array}{c}54 \\
(29.35)\end{array}$ & $\begin{array}{c}60 \\
(32.61)\end{array}$ & $\begin{array}{c}16 \\
(8.70)\end{array}$ \\
\hline $\begin{array}{l}\text { 15. *You touch hand or parts of other people's body } \\
\text { from activities of your daily life }\end{array}$ & $\begin{array}{c}11 \\
(5.98)\end{array}$ & $\begin{array}{c}30 \\
(16.30)\end{array}$ & $\begin{array}{c}43 \\
(23.37)\end{array}$ & $\begin{array}{c}61 \\
(33.15)\end{array}$ & $\begin{array}{c}39 \\
(21.20)\end{array}$ \\
\hline 16. You have sufficient amount of meals each day & $\begin{array}{c}67 \\
(36.41)\end{array}$ & $\begin{array}{c}52 \\
(28.26)\end{array}$ & $\begin{array}{c}40 \\
(21.74)\end{array}$ & $\begin{array}{c}24 \\
(13.04)\end{array}$ & $\begin{array}{c}1 \\
(0.54)\end{array}$ \\
\hline 17. You take useful meals of the 5 food groups & $\begin{array}{c}51 \\
(27.72)\end{array}$ & $\begin{array}{c}54 \\
(29.35)\end{array}$ & $\begin{array}{c}53 \\
(28.80)\end{array}$ & $\begin{array}{c}25 \\
(13.59)\end{array}$ & $\begin{array}{c}1 \\
(0.54)\end{array}$ \\
\hline 18. You eat hot meal or newly cooked food & $\begin{array}{c}69 \\
(37.50)\end{array}$ & $\begin{array}{c}61 \\
(33.15)\end{array}$ & $\begin{array}{c}41 \\
(22.28)\end{array}$ & $\begin{array}{c}13 \\
(7.07)\end{array}$ & $\begin{array}{c}0 \\
(0.00)\end{array}$ \\
\hline $\begin{array}{l}\text { 19. *You buy or eat food that sellers directly touch or } \\
\text { take hold by their hand }\end{array}$ & $\begin{array}{c}14 \\
(7.61)\end{array}$ & $\begin{array}{c}23 \\
(12.50)\end{array}$ & $\begin{array}{c}53 \\
(28.80)\end{array}$ & $\begin{array}{c}45 \\
(24.46)\end{array}$ & $\begin{array}{c}49 \\
(26.63)\end{array}$ \\
\hline 20. You take a rest by sleeping at least $8 \mathrm{~h}$ a day & $\begin{array}{c}20 \\
(10.87)\end{array}$ & $\begin{array}{c}42 \\
(22.83)\end{array}$ & $\begin{array}{c}61 \\
(33.15)\end{array}$ & $\begin{array}{c}53 \\
(28.80)\end{array}$ & $\begin{array}{c}9 \\
(4.35)\end{array}$ \\
\hline 21. You exercise to keep fit and strong & $\begin{array}{c}10 \\
(5.43)\end{array}$ & $\begin{array}{c}29 \\
(15.76)\end{array}$ & $\begin{array}{c}45 \\
(24.46) \\
\end{array}$ & $\begin{array}{c}79 \\
(42.93)\end{array}$ & $\begin{array}{c}21 \\
(11.41)\end{array}$ \\
\hline
\end{tabular}

*Negative statements 
http://wjst.wu.ac.th

Table 7 COVID-19 prevention behavior level $(n=184)$.

\begin{tabular}{lcc}
\hline \multicolumn{1}{c}{ Prevention behavior level } & Frequency & \% \\
\hline Good $(78.00-105.00$ points $)$ & 86 & 46.74 \\
Moderate $(49.00-77.00$ points $)$ & 95 & 51.63 \\
Poor $(21.00-48.00$ points $)$ & 3 & 1.63 \\
\hline
\end{tabular}

Total score 105.00, max. 97.00, min. 23.00, mean 76.40, S.D. 10.83

Table 8 Association between factors and COVID-19 prevention behavior $(n=184)$.

\begin{tabular}{|c|c|c|c|c|c|c|c|}
\hline \multirow[t]{2}{*}{ Variable } & \multicolumn{4}{|c|}{$\begin{array}{c}\text { COVID-19 prevention behavior level } \\
n(\%)\end{array}$} & \multirow[t]{2}{*}{$x^{2}$} & \multirow[t]{2}{*}{$d f$} & \multirow[t]{2}{*}{$p$-value } \\
\hline & & Iigh & Mo & and low & & & \\
\hline & & & & & & & \\
\hline Male & 4 & $(40.00)$ & 6 & $(60.00)$ & & & \\
\hline Female & 82 & $(47.13)$ & 92 & $(52.87)$ & & & \\
\hline Age & & & & & 6.520 & 1 & 0.014 \\
\hline Lower than 20 years & 23 & $(34.33)$ & 44 & $(65.67)$ & & & \\
\hline 20 years and upper & 63 & $(53.85)$ & 54 & $(46.15)$ & & & \\
\hline Curriculum & & & & & 8.836 & 4 & 0.065 \\
\hline Dip. of P.H. (Pharmacy technique) & 16 & $(48.48)$ & 17 & $(51.52)$ & & & \\
\hline Dip. (Emergency Medical Operation) & 7 & (41.18) & 10 & $(58.82)$ & & & \\
\hline B.P.H. Community Health & 27 & (64.29) & 15 & $(35.71)$ & & & \\
\hline B.P.H. Dental Public Health & 23 & (44.23) & 29 & $(55.77)$ & & & \\
\hline B.TM. & 13 & $(32.50)$ & 27 & $(67.50)$ & & & \\
\hline Curriculum level & & & & & 0.015 & 1 & 0.518 \\
\hline Diploma & 23 & $(46.00)$ & 27 & $(54.00)$ & & & \\
\hline Bachelor & 63 & $(47.01)$ & 71 & $(52.99)$ & & & \\
\hline Year level & & & & & 16.454 & 2 & 0.000 \\
\hline $1^{\text {st }}$ year & 25 & $(31.25)$ & 55 & $(68.75)$ & & & \\
\hline $2^{\text {nd }}$ year & 28 & $(50.91)$ & 27 & (49.09) & & & \\
\hline $3^{\text {rd }}$ year & 33 & $(67.35)$ & 16 & $(32.65)$ & & & \\
\hline Residence & & & & & 1.921 & 1 & 0.117 \\
\hline Dormitory & 68 & $(44.44)$ & 85 & $(55.56)$ & & & \\
\hline Outside & 18 & $(58.06)$ & 13 & (41.94) & & & \\
\hline Home town & & & & & 0.124 & 1 & 0.424 \\
\hline Three southernmost provinces & 26 & $(44.83)$ & 32 & $(55.17)$ & & & \\
\hline Other provinces & 60 & $(47.62)$ & 66 & $(52.38)$ & & & \\
\hline Payment per week* & & & & & 9.408 & 2 & 0.009 \\
\hline Lower than 1,000 Baht & 37 & $(42.05)$ & 51 & $(57.95)$ & & & \\
\hline $1,000-1,500 \mathrm{Baht}$ & 37 & $(45.12)$ & 47 & $(54.88)$ & & & \\
\hline More than $1,500 \mathrm{Baht}$ & 12 & $(85.71)$ & 2 & (14.29) & & & \\
\hline Knowledge level on COVID-19 infection & & & & & 0.623 & 1 & 0.294 \\
\hline Good ( $80 \%$ of score) & 76 & $(45.78)$ & 90 & $(54.22)$ & & & \\
\hline Lower than $80 \%$ & 10 & $(55.56)$ & 8 & (44.44) & & & \\
\hline $\begin{array}{l}\text { Satisfaction level of disease prevention } \\
\text { and control measures }\end{array}$ & & & & & 3.727 & 1 & 0.037 \\
\hline High ( $80 \%$ of score) & 50 & $(53.76)$ & 43 & (46.24) & & & \\
\hline Lower than $80 \%$ & 36 & $(39.56)$ & 55 & $(60.44)$ & & & \\
\hline
\end{tabular}

* Fisher's exact test 
http://wjst.wu.ac.th

Table 8 showed the association between studied variables and participants' behavior on COVID-19 prevention. (Researchers did not show the association between religion factor and COVID-19 prevention behaviors because of sensitive issue). Participants age of 20 years and upper had better COVID-19 prevention behaviors than lower age. Year level of study, participants studied in higher year had more likely better COVID-19 prevention behaviors than lower year. Participants with higher payment had lower better COVID-19 prevention behaviors. The results for their satisfaction level of collage's measures on disease prevention and control found that higher satisfaction had better COVID-19 prevention behaviors.

Health sciences students' knowledge was in good level (90.22 \%), similar to a study of COVID-19 and Iranian medical students; the survey on their related-knowledge and preventive behaviors was found that the average of correct answers of knowledge was $86.96 \%$; and $79.60 \%$ had high level of related knowledge on COVID-19 [17].

The participants' age of 20 years and older had better COVID-19 prevention behaviors than lower age. This finding was similar to a study in Songkhla province that participants' age had an association with disease prevention behaviors [18].

The result on participants' year level was found that higher year level had better COVID-19 prevention behaviors. First-year students had the lowest behavior on COVID-19 prevention. This finding indicated that they had been studying on general education, not health relevant subjects, whereas $2^{\text {nd }}$ year and $3^{\text {rd }}$ year students had more subjects on health. It was similar to a study of health promoting behaviors of undergraduate students that year level had relationship to health promoting behaviors [19].

The participants' weekly payment was closely associated with COVID-19 prevention behaviors. Higher payment had higher COVID-19 prevention behaviors. This finding was not consistent with a study of consumer behavior among university students that their expense had correlated with night entertainment. It may reduce their aware of health prevention [20]. The possible reason in this pandemic period is that they have to paid for chemist and protective equipment in order to protect themselves from COVID-19 infection.

The participants had high satisfaction level of college's measures on COVID-19 prevention and control and had association with COVID-19 prevention behaviors. For any measures of each organization, measures may have influence or affect to stakeholders. When measures were announcement to all for act, supporting system should be provided. Similar to a study investigated support system to all relevant users, in order for health promotion of all colleagues [21]. In addition, a study of the confidence on good governance of leader and board of administrators to conduct the activities for health promotion and prevention was found that organization's personnel gave more cooperation to their institution's measures and policies when they trust in good governance of their leader [22]. Furthermore, a study found that not only values, attitudes, norms, motivation, or perceptions but also the mediation of satisfaction have closely been associated with sustainable behavior [23]

\section{Conclusions}

More than $70 \%$ of the participants had true answer $(70.65-99.46 \%)$ and good knowledge on COVID-19 infection (90.22\%). Half of the participants had high satisfaction level of college's measures on COVID-19 prevention and control (50.54\%). COVID-19 prevention behavior, about $51.63 \%$ had COVID-19 prevention behavior at Moderate level. The association between studied variables and participants' behavior on COVID-19 prevention found that age, year level, payment per week, and satisfaction level of their organization's measures on disease prevention and control were significantly association with COVID-19 prevention behavior $(p$ - value $<0.05)$.

\section{Acknowledgements}

Thanks to the participants for answering the research questionnaires before going back home when college was temporarily closeddue to the period of COVID-19 prevention and control. The authors report no conflicts of interests in relation to this work. 
http://wjst.wu.ac.th

\section{References}

[1] I Ghinai, TD McPherson, JC Hunter, HL Kirking, D Christiansen, K Joshi, R Rubin, S MoralesEstrada, SR Black, M Pacilli, MJ Fricchione, RK Chugh, KA Walblay, NS Ahmed, WC Stoecker, NF Hasan, DP Burdsall, HE Reese, M Wallace, C Wang, D Moeller, J Korpics, SA Novosad, I Benowitz, MW Jacobs, VS Dasari, MT Patel, J Kauerauf, EM Charles, NO Ezike, V Chu, CM Midgley, MA Rolfes, SI Gerber, X Lu, S Lindstrom, JR Verani and JE Layden. First known personto-person transmission of severe acute respiratory syndrome coronavirus 2 (SARS-CoV-2) in the USA. Lancet 2020; 395, 1137-44.

[2] F Zhou, T Yu, R Du, G Fan, Y Liu, Z Liu, J Xiang, Y Wang, B Song, X Gu, L Guan, Y Wei, H Li, $\mathrm{X} \mathrm{Wu}, \mathrm{J} \mathrm{Xu}, \mathrm{S} \mathrm{Tu}, \mathrm{Y}$ Zhang, $\mathrm{H}$ Chen and B Cao. Clinical course and risk factors for mortality of adult inpatients with COVID-19 in Wuhan, China: A retrospective cohort study. Lancet 2020; 395, 1054-62.

[3] C Huang, Y Wang, X Li, L Ren, J Zhao, Y Hu, L Zhang, G Fan, J Xu, X Gu, Z Cheng, T Yu, J Xia, Y Wei, W Wu, X Xie, W Yin, H Li, M Liu, Y Xiao, H Gao, L Guo, J Xie, G Wang, R Jiang, Z Gao, Q Jin, J Wang and B Cao. Clinical features of patients infected with 2019 novel coronavirus in Wuhan, China. Lancet 2020; 395, 497-506.

[4] Z Wu and JM McGoogan. Characteristics of and important lessons from the coronavirus disease 2019 (COVID-19) outbreak in China; Summary of a report of 72,314 cases from the Chinese Center for Disease Control and Prevention. JAMA 2020; 323, 1239-42.

[5] A Remuzzi and G Remuzzi. COVID-19 and Italy: what next? Lancet 2020; 395, 1225-28.

[6] Worldometer. Countries where COVID-19 has spread, Available at: https://www.worldometers.info/coronavirus/countries-where-coronavirus-has-spread, accessed March 2020.

[7] WHO Thailand. Coronavirus disease 2019 (COVID-19). WHO Thailand Situation Report, Available at: https:/www.who.int/docs/default-source/searo/thailand/2020-04-26-tha-sitrep-64covid19-en.pdf?sfvrsn=bdf77b6e 2, accessed March 2020.

[8] Royal Thai Government Gazette. Notifications of the Ministry of Health Other countries where are dangerous areas of communicable diseases B.E.2563. The Communicable Diseases Act. B.E. 2558 (A.D. 2015).

[9] Royal Thai Government Gazette. Notifications of the Ministry of Health Other countries where are dangerous areas of communicable diseases (No.2) B.E.2563. The Communicable Diseases Act. B.E. 2558 (A.D. 2015).

[10] COVID-19 situation - Yala province, Available at: http://www.yala.co.th/covid, accessed April 2020.

[11] Covid-19 Situation Reports. Available at: Center for COVID-19 Situation Administration, accessed April 2020.

[12] Center of COVID-19 Administration: Pattani province, Available at: https://covid.pattani.go.th/announce/detail/41, accessed April 2020.

[13] Covid-19 Situation: Narathiwat, Available at: http://www2.narathiwat.go.th/nara2016/covid, accessed April 2020.

[14] Covid-19 Situation in the South of Thailand, Available at: https://mgronline.com/south/detail/ 9630000044572, accessed April 2020.

[15] RV Krejcie and DW Morgan. Determining sample size for research activities. Educ. Psychol. Meas 1970; 30, 607-10.

[16] BS Bloom. Taxonomy of Education Objective, Handbook I: Cognitive Domain. David Mckay, New York, 1956.

[17] MH Taghrir, R Borazjani and R Shiraly. COVID-19 and Iranian medical students; A survey on their related-knowledge, preventive behaviors and risk perception. Arch. Iran. Med. 2020; 23, 249-54.

[18] K Sukkrajang, B Phraomathurot, Y Phraomathurot, T Rattanakool and S Chenkaow. Knowledge and prevention behavior regarding the dengue of people in the area of responsibility of Ban Tha Sai 
http://wjst.wu.ac.th

Tambon Health Promotion Hospital Ko Yo Songkhla. In: Proceedings of the $7^{\text {th }}$ Hatyai National and International Conference, 2016.

[19] A Purakhom and Y Kaewmahingsa. 2013, Health Promoting Behaviors of Undergraduate Students at Kasetsart University, Kamphaengsaen Campus. Kasetsart University, Kamphaengsaen Campus.

[20] J Promwong, A Ngamvichaikit and Y Thamcharoen. Consumer behavior for pub and night entertainment around campus of university students in Bangkok. J. Grad. Stud. VRU 2018; 12, 2337.

[21] P Jinpon, M Jaroensutasinee and K Jaroensutasinee. Integrated information visualization to support decision making for health promotion in Chonburi, Thailand. Walailak J. Sci. \& Tech. 2019; 8, 55160 .

[22] A Singkun and N Samae. The confidence on good governance of leader and board of administrators to conduct the activities for health promotion and prevention. Res. Dev. Health Syst. J. 2019; 12, 478-86.

[23] M Ertz1 and E Sarigöllü. The Behavior Attitude Relationship and Satisfaction in Proenvironmental Behavior. Environ. Behav. 2019; 51, 1106-32. 